\title{
Fixed points of monotone nonexpansive mappings with a graph
}

\section{Monther Rashed Alfuraidan*}

${ }^{\text {*Correspondence: }}$ monther@kfupm.edu.sa

Department of Mathematics and Statistics, King Fahd University of Petroleum and Minerals, Dhahran, 31261, Saudi Arabia

\begin{abstract}
In this paper, we study the existence of fixed points of monotone nonexpansive mappings defined in Banach spaces endowed with a graph. This work is a continuity of the previous results of Ran and Reurings, Nieto et al., and Jachimsky done for contraction mappings defined in metric spaces endowed with a graph.
\end{abstract}

MSC: Primary 06F30; 46B20; 47E10

Keywords: Banach space; directed graph; fixed point; monotone nonexpansive mappings

\section{Introduction}

Banach's contraction principle [1] is remarkable in its simplicity, yet it is perhaps the most widely applied fixed point theorem in all of analysis. This is because the contractive condition on the mapping is simple and easy to test, because it requires only a complete metric space for its setting, and because it is a powerful result with a wide range of applications, including iterative methods for solving linear, nonlinear, differential, integral, and difference equations. Due to its applications in mathematics and other related disciplines, the Banach contraction principle has been generalized in many directions. Recently a version of this theorem was given in partially ordered metric spaces $[2,3]$ and in metric spaces endowed with a graph [4-6].

In this paper, we study the case of nonexpansive mappings defined in Banach spaces endowed with a graph. Nonexpansive mappings are those which have Lipschitz constant equal to 1 . The fixed point theory for such mappings is rich and varied. It finds many applications in nonlinear functional analysis [7]. It is worth mentioning that such investigation is, to the best of our knowledge, new and was never carried out. This work was inspired by [8].

\section{Graph basic definitions}

The terminology of graph theory instead of partial ordering gives a wider picture and yields interesting generalization of the Banach contraction principle. In this section, we give the basic graph theory definitions and notations which will be used throughout.

Let $G$ be a directed graph (digraph) with a set of vertices $V(G)$ and a set of edges $E(G)$ containing all the loops, i.e., $(x, x) \in E(G)$ for any $x \in V(G)$. We also assume that $G$ has no parallel edges (arcs), and so we can identify $G$ with the pair $(V(G), E(G))$. Our graph theory notations and terminology are standard and can be found in all graph theory books 
such as [9] and [10]. Moreover, we may treat $G$ as a weighted graph (see [10], p.309]) by assigning to each edge the distance between its vertices. By $G^{-1}$ we denote the conversion of a graph $G$, i.e., the graph obtained from $G$ by reversing the direction of edges. Thus we have

$$
E\left(G^{-1}\right)=\{(y, x) \mid(x, y) \in E(G)\} .
$$

A digraph $G$ is called an oriented graph if whenever $(u, v) \in E(G)$, then $(v, u) \notin E(G)$. The letter $\widetilde{G}$ denotes the undirected graph obtained from $G$ by ignoring the direction of edges. Actually, it will be more convenient for us to treat $\widetilde{G}$ as a directed graph for which the set of its edges is symmetric. Under this convention,

$$
E(\widetilde{G})=E(G) \cup E\left(G^{-1}\right) .
$$

We call $\left(V^{\prime}, E^{\prime}\right)$ a subgraph of $G$ if $V^{\prime} \subseteq V(G), E^{\prime} \subseteq E(G)$ and for any edge $(x, y) \in E^{\prime}$, $x, y \in V^{\prime}$.

If $x$ and $y$ are vertices in a graph $G$, then a (directed) path in $G$ from $x$ to $y$ of length $N$ is a sequence $\left(x_{i}\right)_{i=1}^{i=N}$ of $N+1$ vertices such that $x_{0}=x, x_{N}=y$ and $\left(x_{n-1}, x_{n}\right) \in E(G)$ for $i=1, \ldots, N$. A graph $G$ is connected if there is a directed path between any two vertices. $G$ is weakly connected if $\widetilde{G}$ is connected. If $G$ is such that $E(G)$ is symmetric and $x$ is a vertex in $G$, then the subgraph $G_{x}$ consisting of all edges and vertices which are contained in some path beginning at $x$ is called the component of $G$ containing $x$. In this case $V\left(G_{x}\right)=[x]_{G}$, where $[x]_{G}$ is the equivalence class of the following relation $\mathcal{R}$ defined on $V(G)$ by the rule:

$y \mathcal{R} z$ if there is a (directed) path in $G$ from $y$ to $z$.

Clearly $G_{x}$ is connected.

\section{Monotone nonexpansive mappings}

Throughout we assume that $(X,\|\cdot\|)$ is a Banach space and $\tau$ is a Hausdorff topological vector space topology on $X$ which is weaker than the norm topology. Let $C$ be a nonempty, convex and bounded subset of $X$ not reduced to one point. Let $G$ be a directed graph such that $V(G)=C$ and $E(G) \supseteq \Delta$. Assume that $G$-intervals are convex. Recall that a $G$-interval is any of the subsets $[a, \rightarrow)=\{x \in C ;(a, x) \in E(G)\}$ and $(\leftarrow, b]=\{x \in C ;(x, b) \in E(G)\}$ for any $a, b \in C$.

Definition 3.1 Let $C$ be a nonempty subset of $X$. A mapping $T: C \rightarrow C$ is called

(1) $G$-monotone if $(T(x), T(y)) \in E(G)$ whenever $(x, y) \in E(G)$ for any $x, y \in C$;

(2) $G$-monotone nonexpansive if $T$ is $G$-monotone and

$$
\|T(x)-T(y)\| \leq\|x-y\|, \quad \text { whenever }(x, y) \in E(G)
$$

for any $x, y \in C$.

The point $x \in C$ is called a fixed point of $T$ if $T(x)=x$. The set of fixed points of $T$ will be denoted by $\operatorname{Fix}(T)$. 
Remark 3.1 For examples of metric spaces endowed with a graph and G-monotone mappings which are Lipschitzian with respect to the graph, we refer the reader to the examples found in [5].

Let $T: C \rightarrow C$ be a $G$-monotone nonexpansive mapping. Fix $\lambda \in(0,1)$. Let $x_{0} \in C$ be such that $\left(x_{0}, T\left(x_{0}\right)\right) \in E(G)$. Define $x_{1}=\lambda x_{0}+(1-\lambda) T\left(x_{0}\right)$. Since the set $\left[x_{0}, T\left(x_{0}\right)\right]=$ $\left[x_{0}, \rightarrow\right) \cap\left(\leftarrow, T\left(x_{0}\right)\right]$ is convex, then $x_{1} \in\left[x_{0}, T\left(x_{0}\right)\right]$, i.e., $\left(x_{0}, x_{1}\right)$ and $\left(x_{1}, T\left(x_{0}\right)\right)$ are in $E(G)$. Since $T$ is $G$-monotone nonexpansive, we get $\left(T\left(x_{0}\right), T\left(x_{1}\right)\right) \in E(G)$ and

$$
\left\|T\left(x_{1}\right)-T\left(x_{0}\right)\right\| \leq\left\|x_{1}-x_{0}\right\|
$$

By induction we construct a sequence $\left\{x_{n}\right\}$ in $C$ such that the following hold for any $n \geq 0$ :

(i) $x_{n+1}=\lambda x_{n}+(1-\lambda) T\left(x_{n}\right)$,

(ii) $\left(x_{n}, x_{n+1}\right),\left(x_{n}, T\left(x_{n}\right)\right)$ and $\left(T\left(x_{n}\right), T\left(x_{n+1}\right)\right)$ are in $E(G)$,

(iii) $\left\|T\left(x_{n+1}\right)-T\left(x_{n}\right)\right\| \leq\left\|x_{n+1}-x_{n}\right\|$.

Such a sequence is known as Krasnoselskii sequence [11] (see also [12-14]). The following result is found in $[15,16]$.

Proposition 3.1 Under the above assumptions, we have

$$
(1+n \lambda)\left\|T\left(x_{i}\right)-x_{i}\right\| \leq\left\|T\left(x_{i+n}\right)-x_{i}\right\|+(1-\lambda)^{-n}\left(\left\|T\left(x_{i}\right)-x_{i}\right\|-\left\|T\left(x_{i+n}\right)-x_{i+n}\right\|\right)
$$

for any $i, n \in \mathbb{N}$. This inequality implies

$$
\lim _{n \rightarrow+\infty}\left\|x_{n}-T\left(x_{n}\right)\right\|=0
$$

i.e., $\left\{x_{n}\right\}$ is an approximate fixed point sequence of $T$.

The first part of this proposition is easy to prove via an induction argument on the index $i$. As for the second part, note that $\left\{\left\|x_{n}-T\left(x_{n}\right)\right\|\right\}$ is decreasing. Indeed we have $x_{n+1}-x_{n}=(1-\lambda)\left(T\left(x_{n}\right)-x_{n}\right)$ for any $n \geq 0$. Therefore $\left\{\left\|x_{n}-T\left(x_{n}\right)\right\|\right\}$ is decreasing if and only if $\left\{\left\|x_{n+1}-x_{n}\right\|\right\}$ is decreasing, which holds since

$$
\left\|x_{n+2}-x_{n+1}\right\| \leq \lambda\left\|x_{n+1}-x_{n}\right\|+(1-\lambda)\left\|T\left(x_{n+1}\right)-T\left(x_{n}\right)\right\| \leq\left\|x_{n+1}-x_{n}\right\|
$$

for any $n \geq 0$. So if we assume that $\lim _{n \rightarrow+\infty}\left\|x_{n}-T\left(x_{n}\right)\right\|=R>0$, then we let $i \rightarrow+\infty$ in the main inequality to obtain

$$
(1+n \lambda) R \leq \delta(C)
$$

for any $n \in \mathbb{N}$, where $\delta(C)=\operatorname{diam}(C)$. Obviously this is a contradiction since both $\lambda$ and $R$ are not equal to 0 .

Remark 3.2 We may let $\lambda$ change with $n \in \mathbb{N}$. In this case the sequence $\left\{x_{n}\right\}$ is defined by

$$
x_{n+1}=\lambda_{n} x_{n}+\left(1-\lambda_{n}\right) T\left(x_{n}\right), \quad n=0,1, \ldots
$$


Under suitable assumptions on the sequence $\left\{\lambda_{n}\right\}$, we will have the same conclusions as Proposition 3.1, see [17] for more details.

Before we state the main result of this paper, let us recall the definition of $\tau$-Opial condition.

Definition 3.2 $X$ is said to satisfy the $\tau$-Opial condition if whenever any sequence $\left\{y_{n}\right\}$ in $X \tau$-converges to $y$, we have

$$
\limsup _{n \rightarrow+\infty}\left\|y_{n}-y\right\|<\limsup _{n \rightarrow+\infty}\left\|y_{n}-z\right\|
$$

for any $z \in X$ such that $z \neq y$.

Definition 3.3 The triple $(C,\|\cdot\|, G)$ has property $(\mathrm{P})$ if and only if for any sequence $\left\{x_{n}\right\}_{n \in \mathbb{N}}$ in $C$ such that $\left(x_{n}, x_{n+1}\right) \in E(G)$ for any $n \geq 0$, and if a subsequence $\left\{x_{k_{n}}\right\}$ $\tau$-converges to $x$, then $\left(x_{k_{n}}, x\right) \in E(G)$ for all $n$.

Theorem 3.1 Let $X$ be a Banach space which satisfies the $\tau$-Opial condition. Let $C$ be a bounded convex $\tau$-compact nonempty subset of $X$ not reduced to one point. Assume that $(C,\|\cdot\|, G)$ has property $(\mathrm{P})$ and the G-intervals are convex. Let $T: C \rightarrow C$ be a G-monotone nonexpansive mapping. Assume that there exists $x_{0} \in C$ such that $\left(x_{0}, T\left(x_{0}\right)\right) \in E(G)$. Then Thas a fixed point.

Proof Consider the Krasnoselskii sequence $\left\{x_{n}\right\}$, from Proposition 3.1, which starts at $x_{0}$. Since $C$ is $\tau$-compact, then $\left\{x_{n}\right\}$ will have a subsequence $\left\{x_{k_{n}}\right\}$ which $\tau$-converges to some point $\omega \in C$. By properties (ii) and (P), we get $\left(x_{k_{n}}, \omega\right) \in E(G)$ for any $n \in \mathbb{N}$. Consider the type function

$$
r(x)=\limsup _{n \rightarrow+\infty}\left\|x_{k_{n}}-x\right\|, \quad x \in C .
$$

Then Proposition 3.1 implies

$$
\lim _{n \rightarrow+\infty}\left|\left\|x_{k_{n}}-x\right\|-\left\|T\left(x_{k_{n}}\right)-x\right\|\right| \leq \lim _{n \rightarrow+\infty}\left\|x_{k_{n}}-T\left(x_{k_{n}}\right)\right\|=0 .
$$

Hence

$$
\limsup _{n \rightarrow+\infty}\left\|T\left(x_{k_{n}}\right)-x\right\|=\limsup _{n \rightarrow+\infty}\left\|x_{k_{n}}-x\right\|
$$

i.e., $r(x)=\lim \sup _{n \rightarrow+\infty}\left\|T\left(x_{k_{n}}\right)-x\right\|$ for any $x \in C$. In particular we have

$$
r(T(\omega))=\limsup _{n \rightarrow+\infty}\left\|T\left(x_{k_{n}}\right)-T(\omega)\right\| \leq \limsup _{n \rightarrow+\infty}\left\|x_{k_{n}}-\omega\right\|=r(\omega) .
$$

In fact we have $r(T(x)) \leq r(x)$ for any $x \in C$ which is an upper-bound of $\left\{x_{k_{n}}\right\}$. Finally if $X$ satisfies the $\tau$-Opial condition, then we must have $T(\omega)=\omega$, i.e., $\omega$ is a fixed point of $T$. 
Remark 3.3 The existence of $x_{0} \in C$ such that $\left(x_{0}, T\left(x_{0}\right)\right) \in E(G)$ was crucial. Indeed, the Krasnoselskii sequence $\left\{x_{n}\right\}$ will satisfy $\left(x_{n}, x_{n+1}\right) \in E(G)$ for every $n$. However, if $\left(T\left(x_{0}\right), x_{0}\right) \in E(G)$ then we will have $\left(x_{n+1}, x_{n}\right) \in E(G)$ for every $n$. In this case, we need to revise property $(\mathrm{P})$ into property $\left(\mathrm{P}^{*}\right)$ defined as follows:

The triple $(C,\|\cdot\|, G)$ has property $\left(\mathrm{P}^{*}\right)$ if and only if for any sequence $\left\{x_{n}\right\}_{n \in \mathbb{N}}$ in $C$ such that $\left(x_{n+1}, x_{n}\right) \in E(G)$ for any $n \geq 0$, and if a subsequence $\left\{x_{k_{n}}\right\} \tau$-converges to $x$, then $\left(x, x_{k_{n}}\right) \in E(G)$ for all $n$.

The following results are direct consequences of Theorem 3.1.

Corollary 3.1 Let $C$ be a bounded closed convex nonempty subset of $l_{p}, 1<p<+\infty$. Let $\tau$ be the weak topology. Let $G$ be the digraph defined on $l_{p}$ by $\left(\left\{\alpha_{n}\right\},\left\{\beta_{n}\right\}\right) \in E(G)$ iff $\alpha_{n} \leq \beta_{n}$ for any $n \geq 1$. Then any G-monotone nonexpansive mapping $T: C \rightarrow C$ has a fixed point provided there exists a point $x_{0} \in C$ such that $\left(x_{0}, T\left(x_{0}\right)\right) \in E(G)$ or $\left(T\left(x_{0}\right), x_{0}\right) \in E(G)$.

Remark 3.4 The case of $p=1$ is not interesting for the weak-topology since $l_{1}$ is a Schur Banach space. But if we consider the weak*-topology $\sigma\left(l_{1}, c_{0}\right)$ on $l_{1}$ or the pointwise convergence topology, then $l_{1}$ satisfies the weak*-Opial condition. In this case we have a similar conclusion of Corollary 3.1 for $l_{1}$.

Corollary 3.2 Let $C$ be a bounded closed convex nonempty subset of $L_{p}, 1 \leq p<+\infty$. Let $\tau$ be the almost everywhere convergence topology. Let $G$ be the digraph defined on $L_{p}$ by $(f, g) \in E(G)$ if and only if $f(t) \leq g(t)$ almost everywhere. Assume that $C$ is almost everywhere compact. Then any G-monotone nonexpansive mapping $T: C \rightarrow C$ has a fixed point provided there exists a point $f_{0} \in C$ such that $\left(f_{0}, T\left(f_{0}\right)\right) \in E(G)$ or $\left(T\left(f_{0}\right), f_{0}\right) \in E(G)$.

\section{Competing interests}

The author declares that he has no competing interests.

\section{Acknowledgements}

The author acknowledges King Fahd University of Petroleum and Minerals for supporting this research. The author thanks the referees for their valuable suggestions including the reference [5].

Received: 5 January 2015 Accepted: 19 March 2015 Published online: 09 April 2015

References

1. Banach, S: Sur les opérations dans les ensembles abstraits et leur application aux équations intégrales. Fundam. Math. 3, 133-181 (1922)

2. Nieto, JJ, Rodríguez-López, R: Contractive mapping theorems in partially ordered sets and applications to ordinary differential equations. Order 22, 223-239 (2005)

3. Ran, ACM, Reurings, MCB: A fixed point theorem in partially ordered sets and some applications to matrix equations. Proc. Am. Math. Soc. 132, 1435-1443 (2004)

4. Alfuraidan, MR: Remarks on monotone multivalued mappings on a metric space with a graph. Preprint

5. Bojor, F: Fixed point theorems for Reich type contractions on metric spaces with a graph. Nonlinear Anal. 75(9), 3895-3901 (2012)

6. Jachymski, J: The contraction principle for mappings on a metric space with a graph. Proc. Am. Math. Soc. 136, 1359-1373 (2007)

7. Browder, FE: Nonexpansive nonlinear operators in a Banach space. Proc. Natl. Acad. Sci. USA 54, 1041-1044 (1965)

8. Bachar, M, Khamsi, MA: Fixed points of monotone nonexpansive mappings. Preprint

9. Diestel, R: Graph Theory. Springer, New York (2000)

10. Johnsonbaugh, R: Discrete Mathematics. Prentice Hall, New York (1997)

11. Krasnoselskii, MA: Two observations about the method of successive approximations. Usp. Mat. Nauk 10, 123-127 (1955)

12. Berinde, V: Iterative Approximation of Fixed Points, 2nd edn. Lecture Notes in Mathematics, vol. 1912. Springer, Berlin (2007)

13. Chidume, C: Geometric Properties of Banach Spaces and Nonlinear Iterations. Lecture Notes in Mathematics, vol. 1965. Springer, London (2009) 
14. Ishikawa, S: Fixed points and iteration of a nonexpansive mapping in a Banach space. Proc. Am. Math. Soc. 59, 65-71 (1976)

15. Goebel, K, Kirk, WA: Iteration processes for nonexpansive mappings. Contemp. Math. 21, 115-123 (1983)

16. Goebel, K, Kirk, WA: Topics in Metric Fixed Point Theory. Cambridge Stud. Adv. Math., vol. 28. Cambridge University Press, Cambridge (1990)

17. Kirk, WA: Fixed point theory for nonexpansive mappings II. In: Sine, RC (ed.) Fixed Points and Nonexpansive Mappings. Contemporary Mathematics, vol. 18, pp. 121-140 (1983)

Submit your manuscript to a SpringerOpen ${ }^{\odot}$ journal and benefit from:

- Convenient online submission

Rigorous peer review

- Immediate publication on acceptance

- Open access: articles freely available online

- High visibility within the field

- Retaining the copyright to your article 